\title{
La fusion thermonucléaire par laser
}

Michel Decroisette (michel.decroisette@wanadoo.fr)

Conseiller scientifique au CEA-DAM

\section{La fusion par confinement}

inertiel est une des deux

voies vers la fusion contrôlée ;

I'article en rappelle les

principes. La démonstration

de la combustion

d'un mélange de deutérium

et de tritium (DT)

en laboratoire, mettant

en œuvre des lasers

de puissance, est attendue

dans la décennie, grâce à

deux installations en cours

de construction aux États-Unis

et en France.

L'article décrit les principales

caractéristiques du Laser

Mégajoule, situé au CEA

CESTA, près de Bordeaux.
Les réactions nucléaires permettent de dégager de grandes quantités d'énergie, si l'on choisit bien les noyaux atomiques impliqués.

On peut casser de gros noyaux, cela s'appelle la fission : c'est le processus par lequel un noyau lourd et instable, comme l'uranium 235 ou le plutonium 239, se brise en deux noyaux plus légers et plus stables. La masse totale des produits de fission étant inférieure à la masse originelle, cette réaction nucléaire s'accompagne d'un dégagement d'énergie important, selon la célèbre formule d'Albert Einstein : $\Delta \mathrm{E}_{\mathrm{c}}=\Delta \mathrm{m} \cdot \mathrm{c}^{2}$ (où $\Delta \mathrm{E}_{\mathrm{c}}$ désigne l'augmentation d'énergie cinétique des particules, $\Delta \mathrm{m}$ la perte de masse, et c la célérité de la lumière).

À l'autre extrémité du tableau périodique des éléments - du côté des atomes légers se présente une autre possibilité de dégager de l'énergie : la fusion nucléaire (le verbe fusionner étant pris dans le sens d'amalgamer). C'est une réaction au cours de laquelle deux atomes légers entrent en collision et forment un noyau plus lourd, mais de masse inférieure à la somme des masses initiales ; ce défaut de masse se retrouve dans l'énergie cinétique des produits de la réaction (voir encadré).

La fusion nucléaire est toutefois plus difficile à réaliser que la fission, car il faut porter la matière à très haute température (plusieurs dizaines de millions de degrés) où elle est à l'état de plasma (mélange d'ions et d'électrons). Ces conditions se rencontrent dans les étoiles.

Les attraits de la fusion nucléaire comme source d'énergie civile sont incontestables: elle ne produit pas de pollution ni de rejet de gaz à effet de serre, et elle présente un faible impact environnemental en raison de l'absence de déchets radioactifs à vie longue [1]. Le deutérium existe dans l'eau de mer, à une concentration de $33 \mathrm{mg}$ par litre ; le tritium se fabrique aisément à partir du lithium, abondant dans l'écorce terrestre, mais également dans l'eau de mer. On dispose de réserves pour des milliers d'années, et

$>>$

\section{La fusion thermonucléaire}

Plusieurs réactions de fusion peuvent être effectuées à partir de noyaux légers. La plus facile à réaliser est la fusion d'un noyau de deutérium $(\mathrm{D})$ et d'un noyau de tritium $(\mathrm{T})$, qui sont des isotopes de I'hydrogène ; on obtient un noyau d'hélium avec une énergie cinétique de 3,5 MeV et un neutron de $14 \mathrm{MeV}$ (emportant donc $80 \%$ de l'énergie libérée). La réaction s'écrit :

$\mathrm{D}+\mathrm{T} \rightarrow{ }^{4} \mathrm{He}(3,5 \mathrm{MeV})+\mathrm{n}(14,1 \mathrm{MeV})$

La fusion de 1 g de mélange DT dégage 340 G), soit 94000 kWh, c'est-à-dire plus de quatre fois l'énergie par gramme dégagée par réaction de fission.

C'est le physicien britannique J.D. Lawson qui, en 1957, établit la condition pour que le bilan d'une réaction de fusion soit positif : le produit de la densité de particules ( $n$ ) par la durée de la réaction $(\tau)$ doit être supérieur à une certaine valeur $k$ (qui est fonction de la température) :

$$
n \times \tau \geq \mathrm{k}
$$

Dans le cas de la réaction citée, $\mathrm{k}$ vaut $10^{14} \mathrm{~cm}^{-3}$.s pour une température de l'ordre de 200 MK. Cette valeur peut être obtenue avec des plasmas très différents :

- des plasmas de faible densité $\left(n \sim 10^{14} \mathrm{~cm}^{-3}\right)$, mais de durée de vie très longue (en pratique, fonctionnant en continu) ; c'est la voie du confinement magnétique ;

- des plasmas de très haute densité $\left(n \sim 10^{24} \mathrm{~cm}^{-3}\right)$ et de durée de vie très courte $\left(\sim 10^{-9} \mathrm{~s}\right)$; c'est la voie du confinement inertiel. 
>>

l'exploitation industrielle de la fusion permettrait donc de résoudre les problèmes liés à notre approvisionnement énergétique.

La fusion "contrôlée » fait l'objet de travaux intenses depuis la Seconde Guerre mondiale. Aucune installation industrielle n'a encore été réalisée ; la difficulté de mettre en ouvre les conditions requises explique la lenteur des progrès. De grands projets sont toutefois en cours, empruntant deux voies différentes.

\section{Les deux voies de la fusion thermonucléaire}

La première méthode est la Fusion par Confinement Magnétique.

Pour augmenter la durée de la réaction, on confine le plasma dans un champ magnétique. Diverses solutions ont été imaginées, mais les plus prometteuses restent celles où les lignes magnétiques sont des hélices d'axe torique qui, après un grand nombre de tours, engendrent des surfaces magnétiques en forme de tores emboîtés ; ce sont en particulier les "Tokamaks ».

La machine européenne JET (Joint European Torus) est la plus grande, avec le tokamak japonais JT60, des installations de recherche sur la fusion magnétique. Elle est implantée sur le site de Culham, près d'Oxford en Angleterre. En 1997, elle a produit $16 \mathrm{MW}$ de puissance de fusion à partir d'un plasma deutérium-tritium.

La communauté internationale a proposé un projet de très grande envergure, baptisé ITER (International Thermonuclear ExperimentalReactor). Le site de Cadarache, en France, a été choisi pour la construction de ce réacteur expérimental, dont l'objectif est de parvenir à la maîtrise des mécanismes qui régissent le plasma en régime permanent (dégagement énergétique de 400 à $500 \mathrm{MW}$ pendant quatre cents secondes) [2, 3].

La mise en fonctionnement d'ITER est prévue pour 2019. En 2020, devrait débuter la construction d'une nouvelle machine baptisée DEMO, destinée à mettre au point les procédures d'exploitation industrielle d'un réacteur en régime nominal. Ce n'est qu'au début des années 2050 que la construction d'une première usine de production électrique pourrait débuter.

La seconde méthode est la Fusion par Confinement Inertiel [4-7].

L'idée à la base de la Fusion par Confinement Inertiel (FCI) est de faire
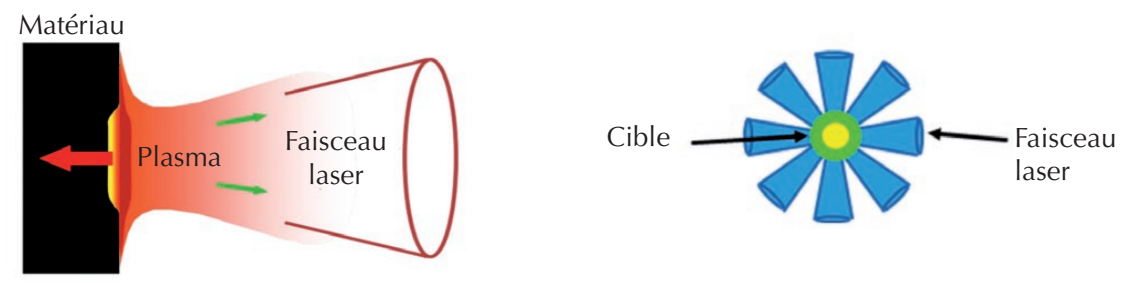

$\mathrm{a}$
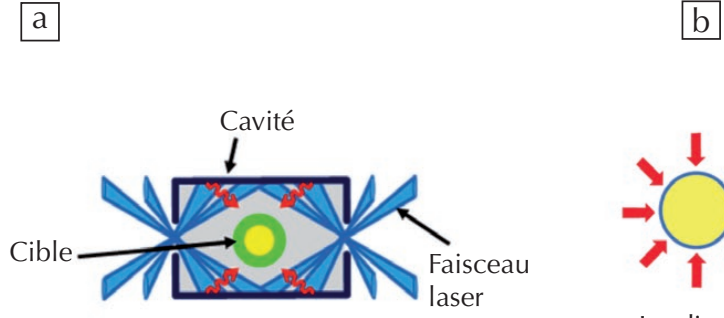

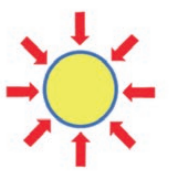

Irradiation

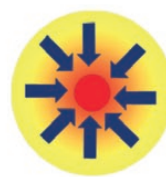

Ignition

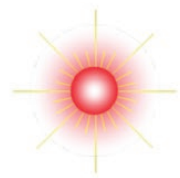

Combustion

1. Principe de la fusion par confinement inertiel par laser. (a) Génération de la surpression. (b) Principe de l'implosion en attaque directe. (c) Principe de l'implosion en attaque indirecte. (d) Principe de l'allumage par point chaud.

imploser une capsule de petite dimension (typiquement $\sim 2 \mathrm{~mm}$ ) contenant un mélange de deutérium et de tritium sous forme cryogénique (en majeure partie solide), en l'irradiant par des faisceaux intenses de rayonnement ou de particules. L'intensité de l'irradiation doit être telle que la densité et la température du mélange comprimé atteignent les valeurs requises par les réactions de fusion, et soient maintenues par inertie le temps que l'énergie thermonucléaire se dégage, avant que la cible se désagrège.

\section{La fusion par confinement inertiel par laser}

C'est l'avènement du laser en 1960 qui fit émerger ce concept, car ce rayonnement cohérent est facilement focalisable. Avec des lasers impulsionnels, on peut atteindre aisément par focalisation du faisceau des densités de puissance très élevées $\left(10^{14} \mathrm{~W} / \mathrm{cm}^{2}\right)$.

\section{Le principe de la FCI}

Si l'on irradie un matériau (en noir sur la figure 1a) sous de telles conditions d'éclairement, on créé un plasma (mélange d'ions et d'électrons) dense et de forte température (plusieurs millions de degrés, zone rouge). Sa détente génère une pression très élevée (de l'ordre de plusieurs millions d'atmosphères). Il en résulte une onde de choc (représentée par la zone claire) qui se propage dans le matériau (flèche rouge). Ce mécanisme est similaire à la propulsion d'une fusée par éjection hors de la tuyère des gaz de combustion. Il peut être exploité pour imploser et comprimer une coquille contenant un mélange fusible. Le principe de l'implosion est illustré sur les figures $1 \mathrm{~b}$ et $1 \mathrm{c}$.

Dans le schéma dit " en attaque directe ", la coquille contenant la cible (de dimension millimétrique) est irradiée par un grand nombre de faisceaux laser dont les impacts sont répartis de sorte que l'interaction soit la plus uniforme possible (fig. 1b).

Dans le schéma en " attaque indirecte ", le rayonnement laser est absorbé dans une enceinte renfermant la cible, et converti (par une succession de processus élémentaires d'absorption, de chauffage et de réémission) en un rayonnement $\mathrm{X}$ qui réalise l'implosion. La cible est donc immergée dans un conteneur, qui agit comme un " four " émettant un rayonnement dit de " corps noir ", auquel on peut associer une température de rayonnement de plusieurs millions de kelvins (fig. 1c). Cette solution est moins intéressante que la précédente sur le plan du transfert d'énergie à la cible, mais assure une meilleure uniformité d'irradiation. C'est l'approche retenue pour l'installation « LMJ » (voir plus loin).

Dans tous les cas, la pression générée entraîne l'implosion de la coquille. Le mélange fusible est porté en son centre à des conditions de densité et de température pour lesquelles la combustion nucléaire se déclenche : 300 g.cm ${ }^{-3}, \sim 70 \mathrm{MK}$. Ce mécanisme est nommé "allumage par point chaud» (fig. 1d). 


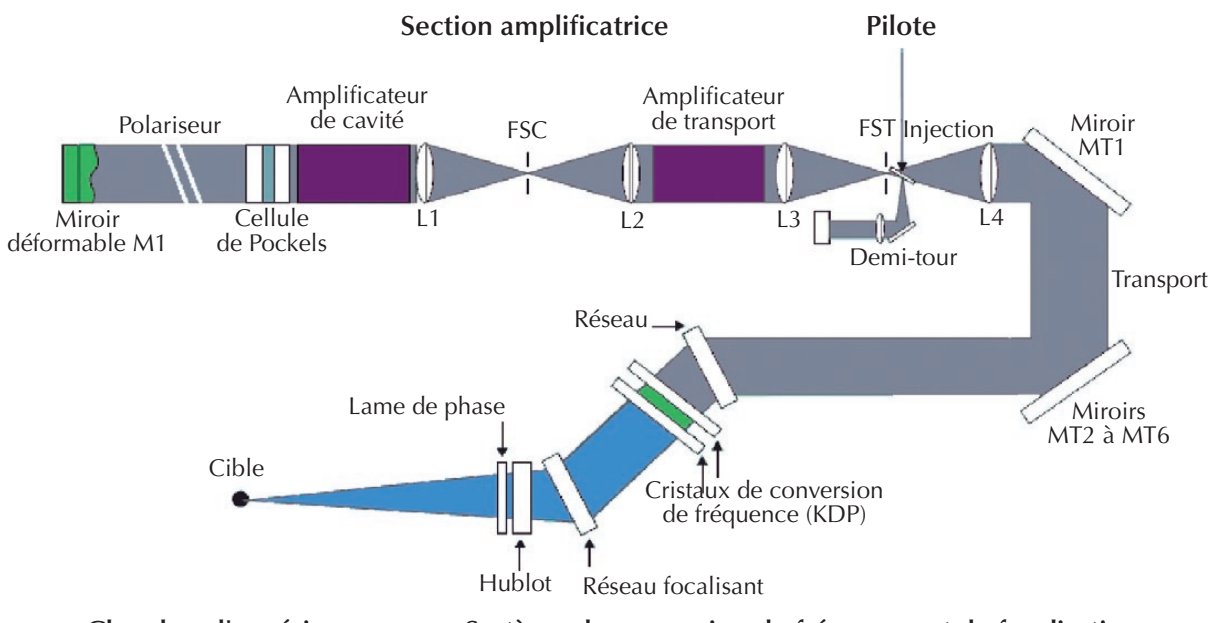

Chambre d'expériences

Système de conversion de fréquence et de focalisation

2. Schéma de la structure d'un faisceau LMJ. Le faisceau issu du pilote se propage vers le miroir M1, revient jusqu'au système "demi-tour ", qui lui fait faire un nouvel aller-retour dans la section amplificatrice ; il en ressort pour aller vers la section de conversion de fréquence et de focalisation. (Document tiré de la référence [3].)

\section{Quelques difficultés}

Atteindre les conditions d'allumage et obtenir la combustion du DT présente des difficultés certaines.

Les travaux théoriques ont montré l'existence d'un seuil (en quantité de DT) à atteindre pour obtenir un gain énergétique (rapport entre l'énergie thermonucléaire dégagée et l'énergie laser déposée sur la cible). Ceci implique une énergie et une puissance minimales du rayonnement laser utilisé pour réaliser l'implosion : il faut disposer de quelques mégajoules délivrés en quelques nanosecondes.

En raison de l'éclairement très élevé auquel on réalise les expériences, des phénomènes non linéaires se développent dans le plasma au cours de l'interaction : ce sont des instabilités qui sont néfastes, car elles s'accompagnent d'une perte d'énergie qui peut être importante, ainsi que de la création d'électrons rapides qui préchauffent le DT, augmentent son entropie et nuisent à la compression.

Des défauts d'éclairement ou de fabrication de la cible pourront induire des instabilités hydrodynamiques qui seront amplifiées, soit pendant la phase d'accélération de la coquille, soit pendant la phase de ralentissement en fin d'implosion, et qui dégraderont l'implosion.

Dans l'optique - lointaine - d'un réacteur, on considère qu'il est nécessaire d'atteindre des gains d'énergie compris entre 30 et 200 pour assurer un fonctionnement satisfaisant au plan économique. L'objectif actuel est de valider le concept en laboratoire dans la prochaine décennie.

\section{Le Laser Mégajoule}

C'est depuis les années 60 que la communauté scientifique s'intéresse à la physique de l'interaction entre un rayonnement laser et la matière. On en a maintenant une assez bonne connaissance. Les progrès réalisés un peu partout dans le monde ont incité deux laboratoires, la Direction des applications militaires du CEA en France, et le Lawrence Livermore National Laboratory aux États-Unis, à construire des installations permettant d'atteindre l'allumage et la combustion du DT : le Laser Mégajoule (LMJ) [8-9] et le National Ignition Facility (NIF) [10-11]. En raison d'un accord officiel de collaboration entre le CEA et le DOE (Departement of Energy), portant sur la mise au point des technologies nécessaires, les conceptions des deux lasers sont proches. Depuis 1999, leurs constructions se poursuivent en parallèle (celle du NIF étant plus avancée que celle du LMJ).

L'objectif du LMJ est de délivrer $2 \mathrm{MJ}$ en $\sim 15$ ns. Il a été conçu sur la base du verre dopé au néodyme comme milieu amplificateur, utilisé depuis plus de trente ans au CEA. Pour obtenir les performances visées avec une marge de sécurité raisonnable, deux cent quarante faisceaux laser ont été prévus. Leur section est carrée ; ils sont regroupés en trente chaînes de huit faisceaux.

Le schéma de base des principaux éléments d'un faisceau est présenté sur la figure 2. Il comporte essentiellement trois tronçons.
Un pilote génère l'impulsion élémentaire (de longueur d'onde $\lambda=1053 \mathrm{~nm}$ ) et fixe toutes les caractéristiques : largeur spectrale, profil temporel, profil spatial de section carrée. Il délivre une énergie ajustable jusqu'à un joule. Un système de miroirs et de lentilles assure le transport jusqu'à la section amplificatrice.

La section amplificatrice comporte deux amplificateurs, chacun constitué de neuf modules (plaques laser) en ligne. Ils sont traversés quatre fois par l'impulsion lumineuse, afin d'accroître le rendement d'extraction de l'énergie déposée par les flashes. Un ensemble de miroirs ( " demi-tour» + M1) associés à des trous de filtrage (FSC et FST) assure ces allers-retours et la sortie du faisceau lumineux. Un miroir déformable (M1) assure la correction de surface d'onde. Les filtrages spatiaux (trous de faible dimension) ont pour objet de limiter les modulations spatiales. Une cellule de Pockels limite la propagation et l'amplification de la lumière parasite.

Dans chaque chaîne, les huit faisceaux sont regroupés, afin d'utiliser au mieux l'énergie délivrée au verre par les lampes flash. C'est la raison du choix de la section carrée des faisceaux lumineux, alors qu'ils sont traditionnellement de section circulaire. Tous les éléments optiques sont installés dans des structures métalliques qui en assurent le positionnement, tout en maintenant un très haut niveau de propreté.

À la sortie de la section amplificatrice, chaque faisceau lumineux, de dimension transversale $\sim 40 \times 40 \mathrm{~cm}^{2}$, délivre $\sim 20 \mathrm{~kJ}$ en $\sim 15 \mathrm{~ns}$. Une succession de miroirs dirige le faisceau lumineux vers le système de conversion de fréquence et de focalisation dans la chambre d'expérience.

Le système de conversion de fréquence, constitué d'un ensemble de deux cristaux de KDP (dihydrogéno-phosphate de potassium), convertit la fréquence de la lumière laser en son harmonique 3 $(\boldsymbol{\lambda}=0,351 \mathrm{~nm})$ pour les besoins de la physique de l'interaction rayonnementmatière (l'efficacité d'absorption par le plasma est plus grande, et limite le développement d'instabilités paramétriques). À cette longueur d'onde, l'énergie délivrée en $\sim 15$ ns est de $\sim 8 \mathrm{~kJ}$. Enfin, la focalisation est réalisée par un réseau de diffraction focalisant. Une lame de phase, qui crée de l'incohérence spatiale, réalise le lissage de la tache focale (diamètre caractéristique : $\sim 700 \mu \mathrm{m})$. 


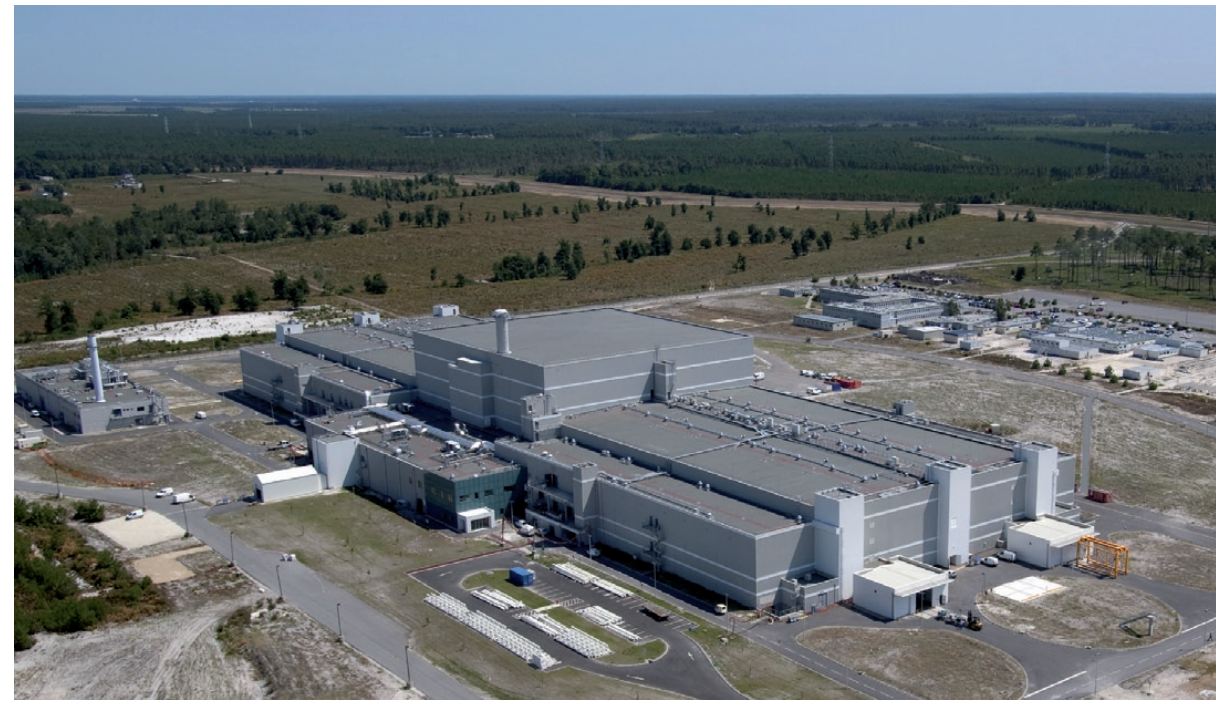

3. Photographie aérienne du Laser Mégajoule en construction au CEA-CESTA. @ (EA G2I Vertigo

\〉

La conception du LMJ présente de nombreuses innovations, comparées aux schémas classiques (multipassages, regroupement des faisceaux, focalisation par réseau, etc.). Il faut également mentionner l'augmentation de la fluence de sortie des chaînes (densité d'énergie en J.cm²) par rapport aux limites habituelles de la tenue au flux des matériaux, en particulier pour les éléments optiques travaillant à la fréquence triple du fondamental.

L'énergie totale délivrée au centre de la chambre d'expériences, à la longueur d'onde $\lambda=0,351 \mu \mathrm{m}$, est de l'ordre de $2 \mathrm{MJ}$. La chambre en aluminium, de dix mètres de diamètre intérieur, supporte un vide meilleur que $10^{-6}$ torr. Elle est placée au centre d'une salle, isolée du reste du bâtiment par deux murs de béton d'un mètre d'épaisseur, qui sont destinés à confiner l'émission neutronique (environ $10^{19}$ neutrons de $14 \mathrm{MeV}$ pour un tir à fort gain). Outre les dispositifs de focalisation et de conversion de fréquence, la chambre reçoit le porte-cible cryogénique $(18 \mathrm{~K})$ ainsi que les systèmes d'insertion de diagnostics, dédiés à la qualification de l'interaction rayonnement-matière et aux mesures des performances de l'implosion.

L'ensemble du système laser et de son banc d'énergie (batterie de condensateurs), de la chambre et des diverses servitudes, est intégré dans un bâtiment de $300 \mathrm{~m}$ de long, $150 \mathrm{~m}$ de large et d'une hauteur maximale de $50 \mathrm{~m}$ (fig. 3).

La construction de cette installation a débuté au Centre CEA du CESTA, près de Bordeaux, en 2003. Les premières expériences sont prévues pour fin 2014.
Le LMJ est financé par le ministère de la Défense, dans le cadre du "Programme Simulation" qui fait suite à l'arrêt des essais nucléaires de la France ; l'objectif est la validation des modèles physiques applicables au domaine thermonucléaire. Mais cet objectif coïncide avec l'étape de démonstration d'un programme visant à la production d'énergie à des fins civiles.

\section{Les perspectives}

En France, le CEA a décidé de construire en avance de phase par rapport au LMJ un prototype, la Ligne d'Intégration Laser (LIL), dont quatre faisceaux sont opérationnels depuis 2002 [12]. Cette installation a été jusqu'à présent utilisée pour effectuer des travaux destinés à valider le concept des cibles à gain retenu pour le LMJ, et réaliser des expériences de physique des plasmas à haute température, dans le cadre de l'ouverture à des équipes académiques.

Le LMJ et le NIF devraient faire la démonstration de la combustion du DT en laboratoire dans le courant de la décennie. Une fois cette démonstration acquise, la question d'un prototype de réacteur se présentera.

C'est dans cet objectif que le Royaume-Uni a présenté un projet baptisé HiPER (High Power laser Energy Research), susceptible de réaliser la fusion avec une énergie de départ plus faible que celle requise par le principe de l'allumage par point chaud, en mettant en œuvre un processus « d'allumage rapide" nécessitant des faisceaux laser annexes [13] ; sa construction pourrait débuter vers les années 2015.
Quelle que soit la voie choisie, un réacteur à fusion présentera deux attraits fondamentaux : aucun risque d'emballement et absence de déchets radioactifs à vie longue. Par rapport à un réacteur à fusion magnétique, un réacteur à fusion inertielle aura l'avantage d'une construction modulaire intéressante pour la maintenance.

De nombreux projets de réacteurs ont été publiés depuis les années soixante-dix. Leur variété résulte de la possibilité d'associer plusieurs types de chambres aux différents "drivers" (sources d'énergie pour imploser la cible) possibles, d'autres solutions que le laser étant envisageables, par exemple des faisceaux de particules.

Comme pour la voie magnétique, d'importants travaux de recherche et développement sont nécessaires pour disposer des divers composants et, en particulier, des matériaux pour réaliser la chambre. Il est prématuré de proposer une échéance pour la réalisation d'un réacteur de démonstration industrielle qui devrait valider le fonctionnement intégré d'un "driver", de la chambre et du système d'injection des cibles, fonctionnant à une fréquence de quelques hertz. On peut l'espérer pour la seconde moitié du $\mathrm{xxI}^{\mathrm{e}}$ siècle.

\section{Références}

1 - La fusion nucléaire: de la recherche fondamentale à la production d'énergie?. Institut de France, Académie des sciences (2007).

2 - R. Arnoux et J. Jacquinot, ITER, Le chemin des étoiles ? Édisud (2006).

3 - Site d'ITER : www.iter.org/fr

4 - R. Dautray et J.P. Watteau, La fusion thermonucléaire inertielle par laser, Eyrolles (1993).

5 - Energy from inertial fusion, International Atomic Energy Agency, Vienne (1995).

6 - M. Decroisette et al., L'énergie de fusion inertielle, Rapport CEA-R-6094 (2005).

7 • B. Bigot et al., Topical issue on "Inertial Confinement Science and Applications", Eur. Phys. J. D 44 (2007) 203-206.

8 - D. Besnard, "The megajoule laser program - ignition at hand”, Eur. Phys. J. D 44 (2007) 207-213.

9 - Site du projet Laser Megajoule : www-Imj.cea.fr

10 - E.I. Moses et al., "The national ignition facility path to ignition in the laboratory", Eur. Phys. J. D 44 (2007) 215-218.

11 - Site du NIF : http://lasers.IInl.gov/

12 • "La Ligne d'Intégration Laser », CHOCS - Revue scientifique et technique de la Direction des applications militaires du CEA, n 29 (2004).

13 - Site du projet HiPER : www.hiper-laser.org 